\title{
A New Cooperative Anomaly Detection Method for Stacker Running Track of Automated Storage and Retrieval System in Industrial Environment
}

\author{
Darong Huang $(\mathbb{D}$, Mengting Lin $(\mathbb{D}$, Lanyan $K e \mathbb{D}$, and Zhenping Deng $(\mathbb{D}$ \\ College of Information Science and Engineering, Chongqing Jiaotong University, Chongqing 400074, China \\ Correspondence should be addressed to Mengting Lin; 622170070012@mails.cqjtu.edu.cn
}

Received 29 March 2018; Revised 28 September 2018; Accepted 25 October 2018; Published 11 November 2018

Academic Editor: Daniel Morinigo-Sotelo

Copyright (c) 2018 Darong Huang et al. This is an open access article distributed under the Creative Commons Attribution License, which permits unrestricted use, distribution, and reproduction in any medium, provided the original work is properly cited.

Considering the complexity and the criticality of the stacker equipment, in order to solve the problem that the stop accuracy of the stacker reduces or even fails to work due to abrasion of the running rail, this paper proposes a cooperative detection method based on Pulse Coupling Neural Network (PCNN) and wavelet transform theory to detect the abnormal points of the stacker running rail in industrial environment by analyzing the variation signals. First of all, considering the fact that the data is mixed up with noises because of the environment at the site and the possibility of the data acquisition equipment breaking down, a noise reduction method for the vibration signal data of stacker is constructed based on PCNN. Then, the basic theory of wavelet transform is introduced and then the rules of judging anomaly points on stackers' running tracks are discussed based on wavelet transform. In addition, a cooperative detection method based on PCNN and wavelet transform theory is carried out based on the spacetime distribution feature of the vibration of the stacker orbits in the industrial environment. Then the rationality of the proposed algorithm is verified by simulation through data provided by State Grid Measuring Center of China. This paper constructs a model of the abnormal point detection of the stackers in an industrial environment. The experimental simulation and example simulation show that the cooperative detection method based on PCNN and wavelet transform theory can effectively detect and locate the anomaly points of the stacker running tracks. The expansibility in engineering applications is promising. Lastly, some conclusions are discussed.

\section{Introduction}

As is known to all, the stacker is a key equipment of automated storage and retrieval system (ASRS) in industrial environment. In practice scene of ASRS, the main function of the stacker is to grab, move, and stack goods from one shelf to another. Thus, the stability of stacker running track will influence the accuracy of grabbing and moving goods in practical engineering. As one of the key components of the stacker equipment, the track of the stacker is divided into upper and lower track. In fact, the stacking machine would wear, crack, sag, and bulge by long time running. And these defects will reduce the accuracy of the stacking machine. If the field engineer cannot detect and repair the defect as soon as possible, they may even cause the whole equipment to be worn and shut down. So, how to detect the anomaly point of stacker running rail is very important for safety performance of the whole system.

In anomaly detection, domestic and foreign researchers have made a lot of progress in all aspects. Since the 1980s, the anomaly detection problem has been widely researched in the field of statistics. For examples, [1] presented an anomaly detection algorithm based on statistical theory. Unfortunately, practice has proved that the computing results of the statistical method are not ideal, because the distribution characteristics of the data must be known in advance. Obviously, the shortage limits the application scope of the method. To solve the defect, some improved detection algorithms were put forward combining with distance in $[2,3]$. The obvious characteristic of the improved algorithm is required to have 
a lot of domain knowledge of real systems or equipment. However, it is known to us all that the running performance of real systems or equipment is affected by various factors. Therefore, it is difficult to determine the input parameters of the algorithm. In other words, it has some disadvantages such as instability and high algorithm complexity. Thus, once the testing data is incomplete, it is difficult to determine the degree of abnormal [4]. In particular, when the data has features such as high-dimensional and sparseness, the performance of the algorithm is very poor. In order to solve the problem caused by sparse data, Rastogi and Ramaswamy proposed an improved algorithm based on density in [5]. The method can avoid the limitation of the dependence of distance to a certain context. To better handle testing data to find the abnormal data point, some detecting ideas based on depth continue to appear. For instance, some researchers presented the anomaly detection algorithm based on depth to mark each record as a point in the $k$ dimension space. In fact, the typical DEEPLOC algorithm was proposed by Struyf and Rousseeuw in [6]. One big advantage of the algorithm is that the depth value of each point may be given according to the definition of depth. The detection rule is that a record with a smaller depth is more likely to be an anomaly point than a larger one. Thus, once the data set is organized by the depth value, the algorithm only needs to carry out the outlier detection on the lower layer of the depth value. Meanwhile, the outlier detection is not required in the record on a layer with a large depth value.

Furthermore, Zhou S and Xu W have constructed the local anomaly detection algorithm based on the deviation in [7]. The algorithm and rule may solve the problem that the existing local anomaly detection algorithms do not perform data object partition. But in real running process of the systems, the high dimensional test data would lead to increased computational complexity [8]. So, the trick to find out the abnormal point from high-dimension data set is to reduce its dimensionality. Aiming at the high dimensionality of the data set, some researchers introduced the concept of local projection scoring (LPS) and proposed an efficient abnormal point detection method of high-dimensional data in [9]. Of course, some new algorithms (for instance, neural network [10] and clustering algorithm [11]) continue to be introduced and presented to detect and monitor the abnormal points of test data. The simulation results show that these models and methods are effective in real engineering and application.

In addition, some scholars had discussed the anomaly detection in the frequency domain. For instance, to overcome the disadvantages that the window size does not change with frequency, the wavelet transform theory is usually introduced to compensate for the localization defects of the short-time Fourier transform [12]. The characteristics of multiresolution (also called multiscale) can be used to observe signal gradually from coarse to fine. The detection and observational modes are favorable for detecting singularities of the signal step by step. So, the wavelet theorem is also an ideal signal time-frequency analysis and processing tools. Among them, the most representative algorithm is the time series anomaly detection method based on wavelet transform proposed in [12].
Although the engineers and scholars have made a lot of achievements in the field of the detection of anomaly spot, the identifying and detection of abnormal data is still in its infancy of research for the stacker of ASRS. The practice application results show that there are still some problems in the methods of safety supervisory of the stacker of ASRS. As is known to all, the running performance of stacker is affected by various factors such as running environment, structural characteristics, and the optimal goal of the whole system. Hence, how to implement the detection of the abnormal points is the kernel problem of safety maintenance of stacker in ASRS. At present, there are some research achievements about the detection of a stacker's running performance. But the research is mainly focused on the structure analysis or the design of the system, and few people have done their researches from the perspective of abnormal point's detection [13]. In recent years, many new algorithms are presented and proposed based on Internet, OPC, and the fault tree to analyze the data set acquired and measured from the running state of the stacker in [14]. In engineering application, the detecting ability of the remote fault of stacker may meet the desired purpose using the proposed algorithm presented in $[15,16]$. Meanwhile, Kai Zhang and his coauthors have discussed and analyzed the monitoring method based on the multimode and multivariate statistics to monitor the running state of stacker crane in $[17,18]$. Experimental results show that the anomaly detection method is effective.

However, because the running state of stacker is influenced by the state of the stacker's component, the scene environment, the data acquisition equipment, and so on, the data set acquired from the system will contain a lot of noise. Obviously, the noise of data will reduce the accuracy of abnormal point's judgment. Therefore, deleting and cleaning the noise from testing data is necessary to implement and accomplish the monitoring of running state of whole stacker system. To restrain the interferences of strong background noise, Huang D.R. and his coauthors have constructed a cooperated denoising algorithm for rolling bearing of stacker in [19]. The simulation results have verified the effectiveness of health monitoring of ASRS. Notice that, in the previous scenario, the actual monitoring data obtained from the real stacker is the aliasing vibration signal. So, how to accomplish the separation of multivibration signal is a difficult problem in vibration process. If the method is reasonable, the detecting accuracy of stacker's running abnormal points will be greatly improved.

Based on the analysis and the thesis above, to ensure the effectiveness of the incomplete data processing of real system, it is necessary to construct and design a cooperative anomaly detection algorithm so that the abnormal spot can be detected and located as quickly as possible in on-site industrial environment. Notice that the timing of the real time data process is vital in the industrial environment, and then the data signal needs to be treated from coarse to fine as soon as possible. On the basis, the Pulse Coupling Neural Network (PCNN) presented in $[20,21]$ is introduced to denoise, because it has the advantage that the industrial data process does not depend on precise mathematics model. Meanwhile, due to the fact that the wavelet transform may complete the itemization 
of the data collection, it is introduced to construct and establish the abnormal point detecting algorithm to locate the stacker's defect through the pure data processed by PCNN.

Hence, the rest of this paper will discuss the details of the algorithm and thesis. The layout of the rest of the paper is organized as follows: Section 2 will introduce the basic concepts of PCNN and the modified PCNN is introduced to construct the data denoising model. In Section 3, in order to locate the anomaly points, the wavelet transform is introduced as the anomaly detection and location algorithm. Also, the cooperative anomaly judgment algorithm and rule for anomaly detection will be discussed in detail combined with modified PCNN and wavelet transform. In addition, the algorithm flow chart is also drawn. Later, the contrast experiments and numerical simulation to detect the abnormal point of stacker running track are carried out to verify the effect of the algorithm and rule using data provided by State Grid Measuring Center of China. Finally, some conclusions and the directions for future engineering application are discussed according to the real stacker running track of ASRS in industrial environment.

\section{Improved PCNN Denoising Model and Algorithm for Vibration Signals of Stacker Running Track}

In a real working condition, the actual vibration signals measured from this system will be unavoidably affected by many complicated environmental factors. Obviously, the data package usually includes strong noises. So, to guarantee the effectiveness of anomaly detection for stacker running track, a reasonable data preprocessing procedure is very crucial to eliminate the noises that are contained in the dataset. In this context, constructing an effective denoising model and algorithm to process the original signal is of great theoretical and practical significance for the condition monitoring of the stacker running track.

However, most industrial monitoring and control applications require high performance, timeliness, and reliability. Then, most administrators and engineers hope to effectively operate the system without knowing the accurate model. Based on this thesis, the PCNN will be introduced later. On this basis, an improved PCNN denoising model and algorithm are analyzed and designed according to the actual situation to ensure the timeliness and stability of the performance of the stacker running track.

2.1. Basic Theory of PCNN. As we all know, PCNN is presented by Eckhorn based on the observed synchronous pulse transmission after the experiments of the cerebral cortex of the animals [22]. In fact, due to its scale invariance, rotation invariance, intensity invariance, distortion invariant, and other characteristics, PCNN is widely used in image smoothing, image segmentation, image edge detection, image fusion, optimal solution, and so on. Moreover, in theory, the PCNN model has similar group neurons synchronization release pulse characteristic and the accurate model is not needed to parse the structure of dataset. Thus, the engineers not only reveal the inherent ability of PCNN, but also explore the application of PCNN in the signal denoising. So, the basic concept of PCNN will be introduced in the next context.

According to [22], the PCNN model can be expressed by the following equation:

$$
\begin{aligned}
& F_{i j}[n]=e^{-\tau_{F_{i j}}} F_{i j}[n-1]+V_{F} \sum_{k, l} M_{i j k l} Y_{k l}[n-1]+S_{i j} \\
& L_{i j}[n]=e^{-\tau_{L_{i j}}} L_{i j}[n-1]+V_{L} \sum_{k, l} W_{i j k l} Y_{k l}[n-1] \\
& U_{i j}[n]=F_{i j}[n]\left(1+\beta L_{i j}[n]\right) \\
& \theta_{i j}[n]=e^{-\tau_{\theta_{i j}}} \theta_{i j}[n-1]+V_{\theta} Y_{i j}[n-1] \\
& Y_{i j}[n]= \begin{cases}1 & U_{i j}[n]>\theta_{i j}[n] \\
0 & U_{i j}[n] \leq \theta_{i j}[n]\end{cases}
\end{aligned}
$$

where $F_{i j}[n]$ denotes the $n$th feedback input for the $(i, j)$ neuron; $S_{i j}$, $U_{i j}$, and $Y_{i j}$ represent the external stimulation, internal behavior, and output of neuron $N_{i j}$, respectively; $L_{i j}, F_{i j}$ are the two input channels for the link domain and the feedback domain of the neuron $N_{i j} ; M$ and $W$ are the connection weight coefficient matrix of feedback domain and link domain; $\theta_{i j}$ and $V_{\theta}$ are the output and threshold amplification factor and the variable threshold function; $\beta$ denotes synaptic link coefficient; $\tau_{L}, \tau_{F}, \tau_{\theta}$ are the time attenuation constants of link domain, feedback domain, and variable threshold function, respectively.

From the perspective of simulation, the PCNN neuron consists of three parts: receiving domain, modulation domain, and pulse generation domain. In real application, PCNN has the advantage that the data processing does not depend on precise mathematics model. That is to say, in the pretreatment of denoising, once the network interface of PCNN receives the input signal, the receiving field transmits it through two channels, $F$ and $L$. The impulse response function of $F$ channel changes slowly with time compared to $L$ channel. The modulation part combines the signal $L_{i j}$ from channel $L$ with an offset and multiplies the signal $F_{i j}$ from channel $F$ to generate the internal signal $U_{i j}$. Then, $\theta_{i j}$ and $U_{i j}$ will be compared to control the firing of signal neurons. If $\theta_{i j}>U_{i j}$, the neurons will be activated. Otherwise, the neurons may be deleted from the structure of PCNN.

According to the link coefficient of $L$ channel and $F$ channel, PCNN can be divided into two cases: coupled and uncoupled. When $\beta=0$, each neuron was separately operated and unaffected by the pulse output of other neurons around it. In addition, considering the friendly interface for end users, the basic structure of PCNN is shown as Figure 1.

Obviously, the original dataset may be used to identify the character of noises by training the PCNN network. Of course, the noise-polluted data may be cleaned through analyzing the PCNN firing matrix and then the noises may also be filtered from the original dataset. In general, the engineers may test the abnormal point in a long data sequence to better locate the defect of stacker running track. 


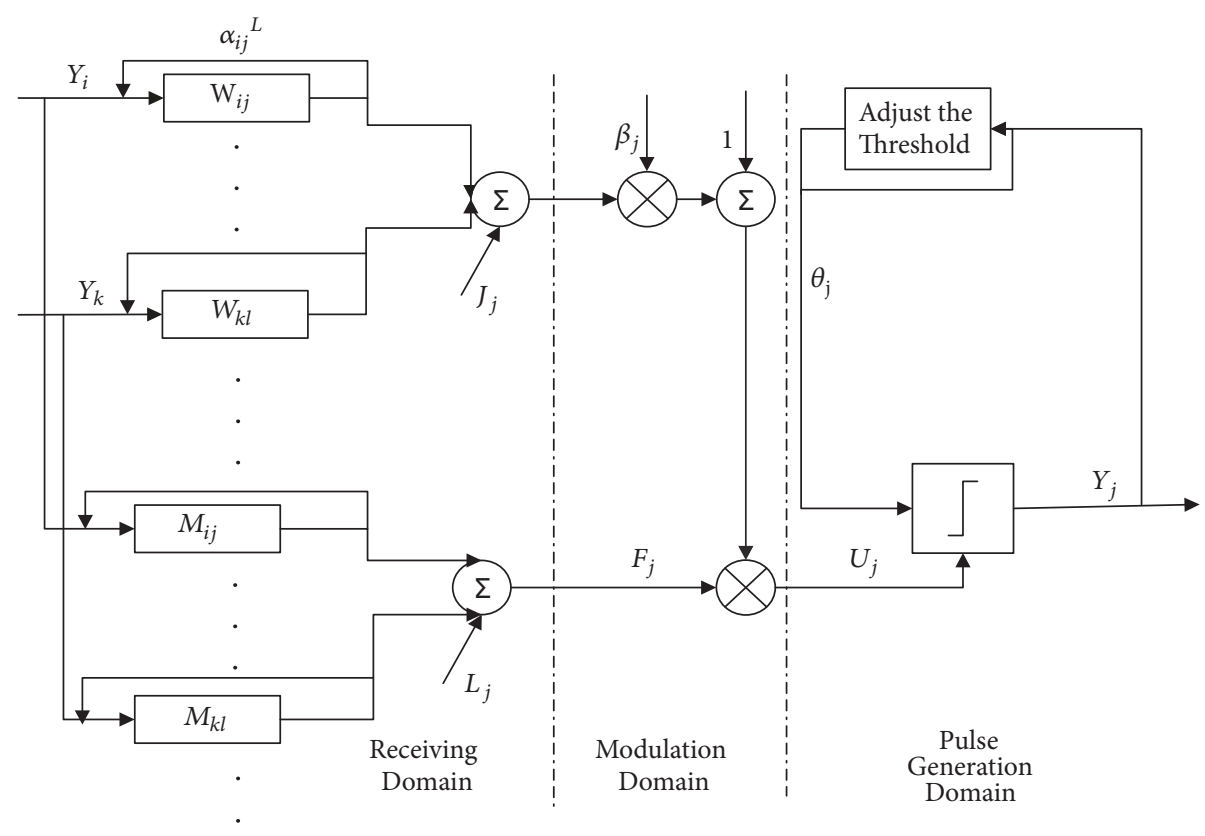

FIGURE 1: Basic structure of PCNN model diagram.

Unfortunately, although the basic structure of PCNN can clean the real dataset mixed with noise, the complexity of this topological structure can cause various issues that negatively impacts the engineer's operation play experience. In real scenario of ASRS, the difficulty in condition monitoring is to ensure the timeliness of locating the abnormal position of stacker running track. In particular, because the running process of stacker is complex and changeable, the original dataset signified the running state of the stacker including lots of noise. From an operational perspective, the traditional denoising method based on nominal model may hardly achieve the expected denoising performance. So, it is necessary to simplify network structure to meet the actual demand and it is a nontrivial problem to construct an improved denoising algorithm based on the existing PCNN model to meet the actual needs.

\subsection{Modified PCNN Denoising Model for Vibration Signals} of Stacker Running Track. In the industrial scenario, due to very poor measurement environment, the quality of the measurement signals may be affected by all kinds of factors. Meanwhile, the influential factors are connected with each other in vibration signals of stacker running track and they also have indirect effects. How to solve the coupling relations within the limits of real time control is very important to denoise the original dataset. In fact, considering that the basic topological structure of PCNN has the excessive parameters and the implementation is more complicated, it is not widely used in practice compared to the modified PCNN model. For a handy operation or statistics, the modified uncoupled model proposed in [21] is adopted to simplify the denoising process.
According to [21], the simplified PCNN model can be expressed by the following equation:

$$
\begin{aligned}
& F_{i j}[n]=S_{i j}[n] \\
& Y_{i j}[n]= \begin{cases}1 & F_{i j}[n]>\theta_{i j}[n] \\
0 & F_{i j}[n] \leq \theta_{i j}[n]\end{cases} \\
& \theta_{i j}[n]= \begin{cases}V_{\theta} & Y_{i j}[n]=1 \\
e^{-\tau_{\theta}} \theta_{i j}[n-1] & Y_{i j}[n]=0\end{cases} \\
& B_{i j}[n]= \begin{cases}1 & Y_{i j}[n]=1 \\
0 & Y_{i j}[n]=0\end{cases} \\
& T_{i j}[n]= \begin{cases}n & Y_{i j}[n]=1 \\
T_{i j}[n-1] & Y_{i j}[n]=0\end{cases}
\end{aligned}
$$

where $F_{i j}[n]$ denotes the nth feedback input for the $(i, j)$ neuron; $S_{i j}$ denotes the external stimulation of neurons $N_{i j}$; $\tau_{\theta}$ denotes time attenuation constants of variable threshold function.

In this model, the signal from the channel $L$ is not accepted by $F_{i j}$. As a result, $F_{i j}$ is only affected by external input. The $B$ matrix records whether the neuron fires at the nth input. And when the neuron fires, it will be assigned a larger threshold, so that it will not be fired again in a short time. Conversely, the threshold of the neuron that is not fired will decay over time. Therefore, the $T$ matrix is used to record the time when each neuron is firstly fired. The noise point in the data can be filtered out by the value of $T$ matrix. 


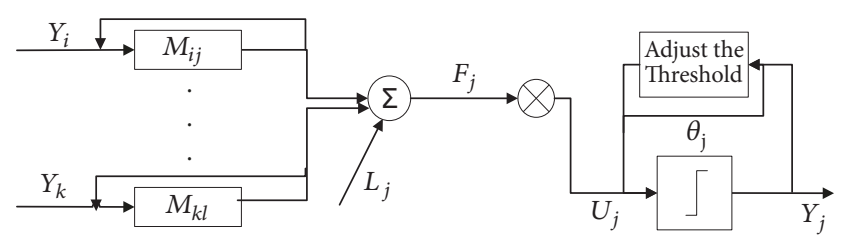

Figure 2: Modified PCNN model diagram.

The modified topological structure of simplified PCNN is shown as Figure 2.

Obviously, the modified model has advantages of less parameters and simple implementation. In other words, the simplified denoising system has operational simplicity and high efficiency for the denoising processing of the raw incoming data. For the sake of applying in industry, the simplified denoising process is more applicable. Thus, we can make use of the modified denoising model to denoise the vibration signals of stacker running track and finally realize the recognition of anomaly points.

\section{Design and Analysis of Cooperative Anomaly Detection Method and Algorithm for Stacker Running Track of ASRS}

In the above section, one valid dimensional data cleaning model and framework was discussed and analyzed. Once the original dataset measured from the track running system was processed by the modified PCNN, the errors, noises, or missing data that are contained in the dataset should be eliminated and removed. That is to say, the output dataset may be used to accurately locate the anomalous point for the stacker running track of ASRS. Obviously, the key stage of locating the abnormal defect of stacker running track is to find a reasonable model. The engineering practice shows that the energy of induction signal induced by the abnormal point of the stacker running track concentrates on the high frequency band. Of course, the fact has also provided a basis for determining anomaly point of stacker running stacker. Based on this, it is imperative to find a reasonable way to decompose the output dataset of modified PCNN into two parts: low frequency part and high frequency part.

Simultaneously, because the wavelet transform developing since 80 's of last century has specific property of time-frequency localization, the method is especially fit for analyzing and detecting local signal mutation for the stacker running track of ASRS. Next step, the cooperative anomaly detection model of stacker running track will be constructed and analyzed based on wavelet transform.

3.1. Cooperative Anomaly Detection Method Based Wavelet Transform. In our experiments, the main feature of signal mutation is that the signal has local changes in time and space. To guarantee the accuracy of locating and identifying the defects of stacker running track, we have tried to design and construct a cooperative detection method combined with wavelet theory. The principle of using wavelet transform to detect the anomalous points is to decompose the signals in different resolutions. When the signal is abrupt, the coefficient gotten by the wavelet transform has a modulus maximum value. Therefore, the location of the outliers can be filtered out through the detection of the maximum modulus point.

Without loss of generality, the basic principle of wavelet transform is clearly defined: Suppose $\phi(t) \in L^{2}(R)$ is a square integral function, if its Fourier transform satisfies the following condition.

$$
\int_{\mathrm{R}}|\Psi(\omega)|^{2}|\omega|^{-1} d \omega<+\infty
$$

where $\psi(\omega)$ is wavelet transform of $\phi(t)$ and $\phi(t)$ is a wavelet function or base wavelet.

In fact, when the output dataset of modified PCNN was decomposed into high frequency and low frequency, the key factor is to carry the expansion or contraction, and the translation based on the base wavelet. If the expansion or contraction factor is supposed as $a$ and the translation factor is supposed as $b$, then the wavelet function can be rewritten as follows.

$$
\phi_{a, b}(t)=a^{-1 / 2} \phi\left(\frac{t-b}{a}\right) \quad a>0, b \in R
$$

where $a$ denotes the scaling factor (or scale factor) and $b$ is translation factor. And $\phi_{a, b}(t)$ is wavelet base function which is concerned with $a$ and $b$. It can be obtained by scaling or moving the parent function $\phi(t)$.

So, for an arbitrary signal $x(t) \in L^{2}(R)$, the wavelet transform can be expressed by the inner product of the signal and wavelet base function

$$
\begin{aligned}
W_{x}(a, b) & =\left\langle x, \phi_{a, b}(t)\right\rangle \\
& =a^{-1 / 2} \int_{R} x(t) \phi_{a, b}\left(\frac{t-b}{a}\right) d t
\end{aligned}
$$

Notice that the actual measured signals indicating the changes of the running state of the stacker running track are discrete in the stacker running track in ASRS, and the wavelet transform should be rewritten in a discrete form to treat the output dataset provided by modified PCNN. In general, the scaling factor $a$ and the translation factor $b$ are put as follows.

$$
\begin{aligned}
& a=2^{-j}, \\
& b=2^{-j} k
\end{aligned}
$$

Thus, $W_{x}(a, b)$ became a discrete binary wavelet function as follows.

$$
\begin{aligned}
\phi_{j, k} & =2^{j / 2} \phi_{j, k}\left(2^{j} t-k\right) \\
W_{x}(j, k) & =2^{j / 2} \int_{R} x(t) \phi\left(2^{-j} t-k\right) d t
\end{aligned}
$$

where $j, k \in Z$.

Then, the output dataset denoising by modified PCNN may be decomposed into high frequency and low frequency by the following formula.

$$
W_{x}(j, k)=\sum_{j, k=-\infty}^{\infty} x(t) \phi_{j, k}(t)
$$




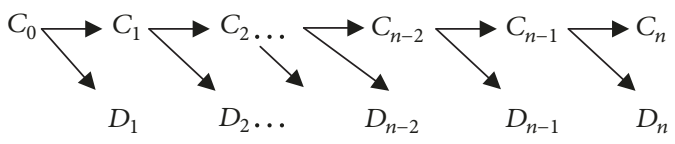

Figure 3: The wavelet decomposing process of output dataset.

Obviously, the wavelet series information may be used to show the changes of the running state of the stacker track. Moreover, to ensure that the energy of induction signal induced by the abnormal point of the stacker running track concentrates on the high frequency band, we have selected the orthogonal wavelet transform to decompose the output dataset into high frequency and low frequency. The basic decomposing mode is defined as follows.

$$
\begin{aligned}
& C_{j+1}=H * C_{j} \\
& D_{j+1}=D * C_{j}
\end{aligned}
$$

where $C$ is the high frequency part and $D$ is the low frequency part.

In our experiments, if the raw output signal $x(t)$ is described as $C_{0}$, the wavelet decomposing process may be shown as Figure 3.

Obviously, according to the decomposing process in Figure 3, the high frequency band of the dataset, which indicates the energy of induction signal induced by the abnormal point of the stacker running track, can be gotten. To add extra flexibility, the high frequency band is described as $C_{j}(j=0,1, \cdots)$.

In practical project, the low-frequency coefficients reflect the contour of the original signal and the high-frequency coefficients describe the details of the signal. In particular, the singularity in the signals is often caused by a sudden change in frequency domain. For the engineers in health monitoring of stacker running state, it means that the high-frequency coefficients of wavelet transform can highlight singularity and can be used for detection and localization of the defect of the stacker running track. From the angle of engineering application, the damage of stacker running track will cause perturbations of measured signal at damage sites. Moreover, the measurement dataset is an aggregate of the running information, and the singularity of high frequency may depict the character of damage position on stacker running track. In other words, the damage concussion will cause the signal saltation. So, in practical engineering applications, it is very important to design a reasonable judgment rule of anomaly points.

To better locate the defects of stacker running track, the Lipschitz index is used to describe and design the judgment rule of singularity of the dataset. For the simplicity of analysis, the corresponding concept of Lipschitz index was defined as follows.

Definition 1. If there exists a constant $c>0$ and $h$, such that $f(t)$ satisfies the following condition

$$
\left|f\left(t_{0}+h\right)-f\left(t_{0}\right)\right| \leq c|h|^{\alpha}
$$

thus, $\alpha$ is the Lipschitz index of $f(t)$ at $t=t_{0}$. And then if $\alpha<1, f(t)$ is singular at $t=t_{0}$.

In general, the size of Lipschitz index is related to the value of the singularity in actual project; i.e., the more severe the degree of mutation, the steeper the peak of the catastrophic point, and the smaller the singularity index, and vice versa. Based on this thesis, we can define the local singularity of high frequency signals decomposed by wavelet transform as follows.

Definition 2. $\forall x \in \delta x_{0}$, if there exists a constant $K>0$ and $s$, the wavelet function $\phi(x)$ is continuous and differentiable and the wavelet transform has $N$ vanishing moment ( $N$ is a positive integer); i.e.,

$$
|W f(s, x)| \leq K s^{\alpha}
$$

Thus, $\alpha$ is the local singularity at $x_{0}$.

Notice that wavelet transform is applied to vibration signal analysis of stacker running track to detect the meshing abnormality of track with local defects, and the amplitudes of the vibration single will decrease or increase to some extent. So, by Definition 1, once there exists $\alpha<1$ at one point, the engineers may judge that the position of the stacker running track may be damaged. Thus, the judgment rule of anomaly point is designed as follows.

Rule 1. $\forall x \in \delta x_{0}$, if the wavelet transform coefficient satisfies

$$
|W f(s, x)| \leq\left|W f\left(x, x_{0}\right)\right|
$$

thus, $x_{0}$ may be judged as the abnormal point of the stacker running stack.

Notice that the mutation information of flaws may be accumulated in running process of stacker's track, and the ability of a single high-frequency detail signal to reflect an abnormal point has limitations to locate the defect to stacker running track. To solve and overcome the problem, the product of the high-frequency coefficient, which can amplify the detail signal, is selected as the basis for the final judgment of the abnormal point in our experiments. To satisfy the need of engineering design, the accumulated information of track's flaws can be described by using the following formula.

$$
C=C_{j} \times C_{j_{n-1}} \times C_{j_{n-2}}
$$

where $C$ is the product of high-frequency signal. $C_{j}, C_{j_{n-1}}$, $C_{j_{n-2}}$ are the $n$-order, $n-1$-order, and $n-2$-order highfrequency detail signals at the decomposition scale $n$, respectively.

Then, the basic rule is shown as follows.

Rule 2. If a mutation appears in the product of the highfrequency coefficient, it is an abnormal point.

Through the analysis and rules above, once the output dataset processed by modified PCNN has been decomposed into the low frequency and high frequency components of the signals, the high frequency components can be used to detect and locate the anomaly points of stacker running track. 
3.2. Design and Analysis of Cooperative Anomaly Detection Algorithm Based on Modified PCNN and Wavelet Transform. Based on the above analysis and discussion, combining with the modified PCNN and wavelet transform, the cooperative anomaly detection algorithm of the stacker running track may be designed in detail as below.

Step 1. Normalize the data. Calculate the initial mean square error and initialize the network parameters by (1)-(9).

Step 2. Use the normalized data as input data. End the loop if matrix $B=0$. Get matrix $\mathrm{T}$ when the loop ends.

Step 3. The noise points are determined according to the elements in the matrix, and then filter each data point.

Step 4. Calculate mean square error (MSE), and compare it with the mean square error before, if the mean square error is smaller than the mean square error before, back to Step 2. Input data into that model to continue to reduce noise. Otherwise, output the signal last time as the result.

Step 5. Select wavelet base function and determine the number of layers of wavelet decomposition.

Step 6. The wavelet transform coefficients of each layer are obtained by wavelet decomposition.

Step 7. Multiply the detail signal to amplify the mutation signal.

Step 8. Obtain the location of the outliers according to the location of the signal mutation.

The algorithm flow chart is shown as Figure 4 .

\section{Examples and Analysis}

\subsection{Simulation Examples}

4.1.1. Simulation Results of Noise Reduction Effect of PCNN. To verify the noise reduction effect of modified PCNN, the simulation examples were first used to test the denoising ability of PCNN. In our simulation experiments, the sinusoidal signal was selected and adopted as the input data to train and test PCNN rule and the Gaussian white noise with a mean of zero and a variance of 1 is added to the sinusoidal signal as the experimental signal. The testing signal $x(t)$ was described as follows.

$$
x(t)=\sin (t)+v
$$

where $v$ is the Gaussian white noise, and $v \sim N(0,1)$.

In actual imitative preparation, $v, n$, and $t$ were set up as below, respectively.

$$
\begin{aligned}
v(t) & =\text { normrnd }(0,0.1, n) \\
t & =0: 0.1: 100 \\
n & =\text { length }(\sin (t))
\end{aligned}
$$

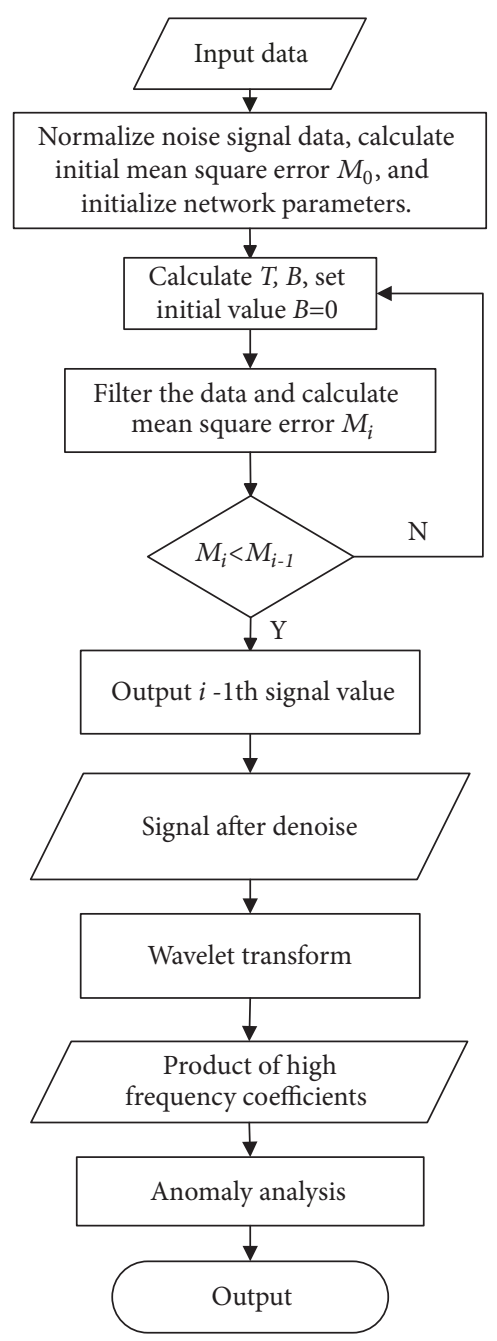

Figure 4: Flow chart of the algorithm.

By training the neural network of PCNN, the denoising results are shown as Figure 5.

Simultaneously, to explain the validity of PCNN denoising algorithm, the wavelet threshold denoising algorithm was used to compare with the presented algorithm in our simulation examples. The effect of wavelet threshold denoising algorithm is shown as Figure 6.

Obviously, although the comparison results between Figures 5 and 6 indicate the differences of the different denoising algorithm, the details of the differences are not obvious and significant. So, we must have a valid index selected to show the differences. To go through this process, the signal-to-noise ratio (SNR) and minimum mean square error (MSE) were used to compare the advantages of proposed denoising algorithm. The basic formulas were shown as follows.

(1) Signal-to-Noise Ratio

$$
S N R=\frac{\widehat{S}_{t}}{v}
$$



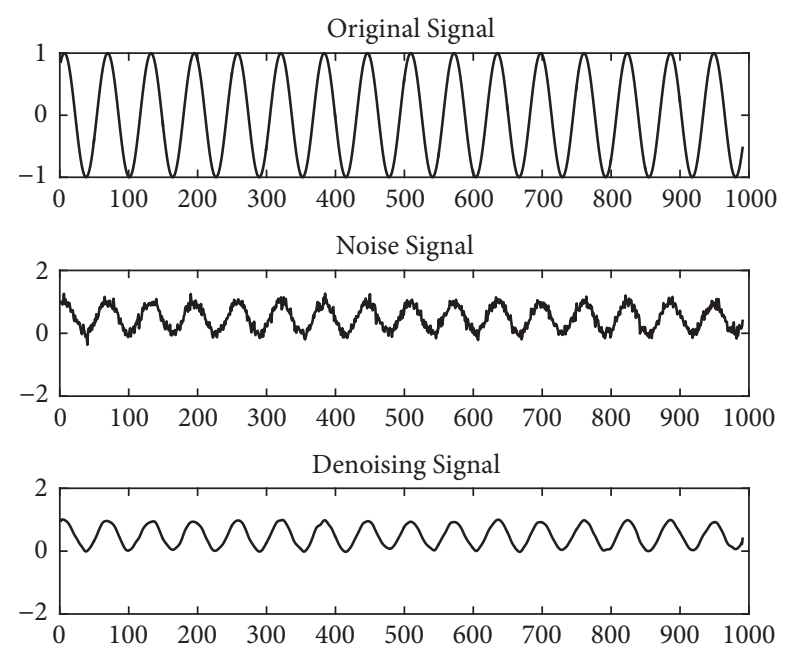

FIGURE 5: PCNN noise reduction results.
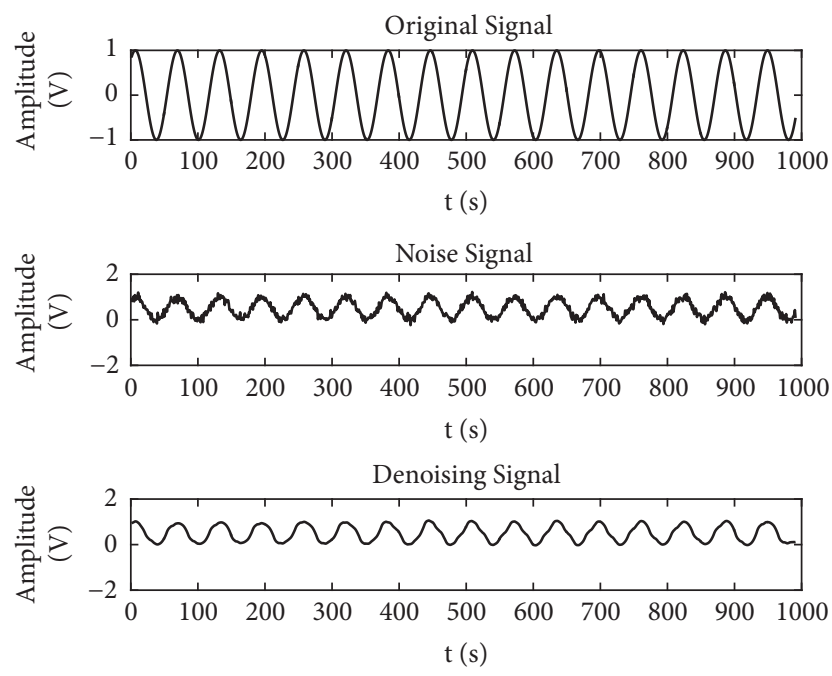

Figure 6: Wavelet threshold denoising results.

TABLE 1: Comparison of wavelet threshold denoising and PCNN noise reduction.

\begin{tabular}{lcc}
\hline & Wavelet denoising & PCNN denoising \\
\hline SNR & 15.15 & 16.47 \\
MSE & 0.37 & 0.36 \\
\hline
\end{tabular}

(2) Mean Square Error

$$
M S E=\frac{1}{n} \sqrt{\sum_{t=1}^{n}\left(S_{t}-\widehat{S}_{t}\right)^{2}}
$$

where $\widehat{S}_{t}$ is denosing signal, $S_{t}$ is the original signal, and $v$ is the noise signal.

By (25) and (26), the corresponding comparison results are shown in Table 1.

It can be seen in Table 1 that PCNN can retain the characteristics of the original signal while getting good noise reduction with higher SNR and lower MSE. So, it means that the denoising effect of modified PCNN is better than wavelet threshold denoising method.

4.1.2. Simulation Results of Anomaly Detection. In order to further verify the validity of cooperative anomaly detection method for singular point in signal dataset, a set of vibration signal with abnormal points is selected to test the effect of presented cooperative anomaly detection algorithm; i.e.,

$$
x(t)= \begin{cases}\sin (0.02 \pi t) & 0 \leq t \leq 400 \\ \sin (0.2 \pi t) & 401 \leq t \leq 600\end{cases}
$$

In our simulation experiments, the simulation results are shown as Figure 7.

In fact, it can be seen from the simulation results that the sudden change of the signal appears at $t=400$ which can be observed from detail coefficients clearly. That indicates that the anomalies appear at $t=400$. It can be concluded that the presented cooperative anomaly detection can mark the location of the abnormal point of the vibration signal clearly. On the other hand, after testing the function, the presented cooperative algorithm may be applied to actual project.

4.2. Real Experiment Examples. Moreover, to further verify the validity of the proposed algorithm, the actual stacking machine vibration signal of ASRS, which has been developed and applied in State Grid Measuring Center of China, was selected to simulate the performance of the anomaly detection algorithm. The prototype systems of the stackers of State Grid Measuring Center of China is shown in Figure 8.

As can be seen in Figure 8, the main function is to grab, move, and stack goods from one goods shift to another. In actual project, the degree of unevenness of flaws (such as rusts, crack, the concavo-convex fluctuation, or any welding defects) will directly influence the safety and effectiveness of whole ASRS. In real engineering, the test rig of stacker running track is shown as Figure 9.

Figures 8 and 9 demonstrate that the crucial equipment of ASRS is the stacker running track. And then the operation track systems of the stacker contain the suspension transporting system (i.e., upper track) and road tracks (i.e., lower tracks). Thus, if outliers are presented and detected within the dataset, the defects of the stacker running track may be located and identified according to the precise geospatial data.

In real application, the sampling time is from March to April of 2018. The size of the sampling data source measured from stacker running track is 7370 . The data may contain noise because of the interference of the site environment and collecting data equipment and other reasons. To the Management Department of State Grid Measuring Center of China, the engineers especially want to find the anomaly points and locate the defects of stacker running track from the experimental data. If the engineers can do this, the whole security of ASRS may be better ensured. So, the simulation results between the original signal and compounded signal with noise caused by the defects of stacker's running track 


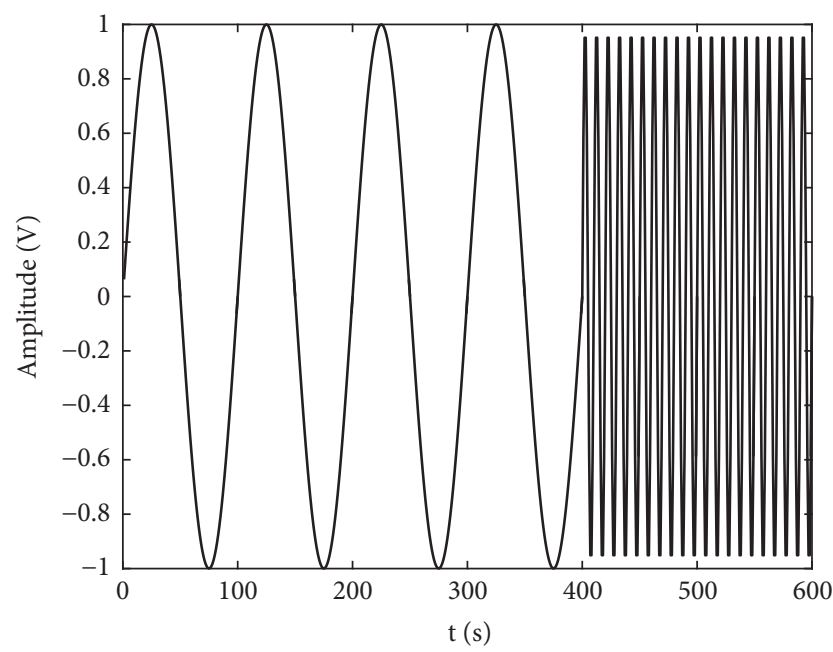

(a) The original signal containing the exception point
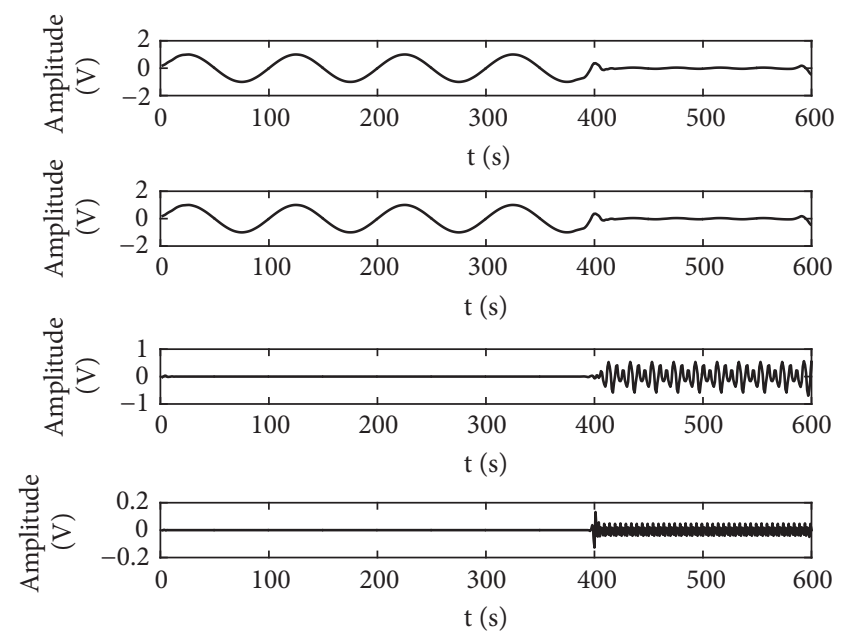

(b) Wavelet decomposition result

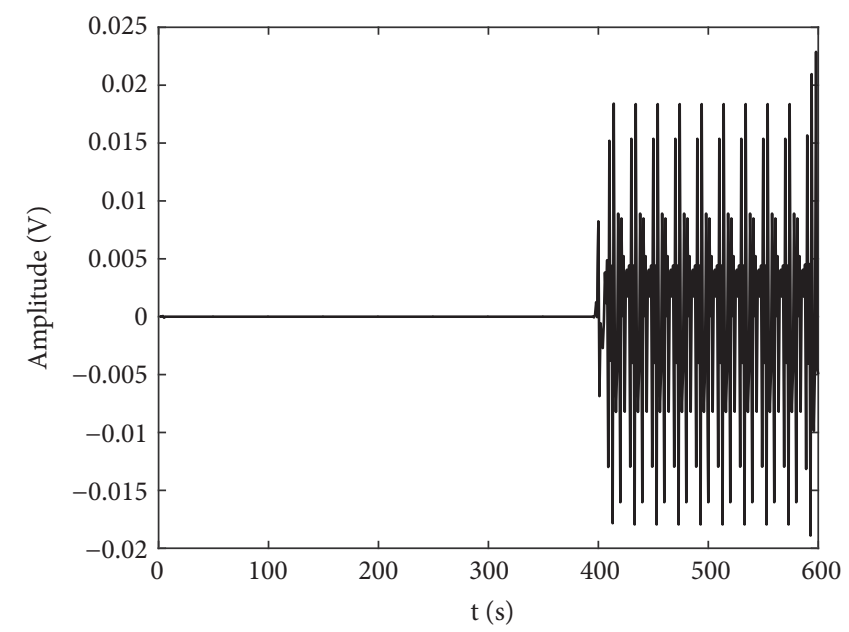

(c) Abnormal point detection results

FIGURE 7: Simulation result of cooperative anomaly extraction.

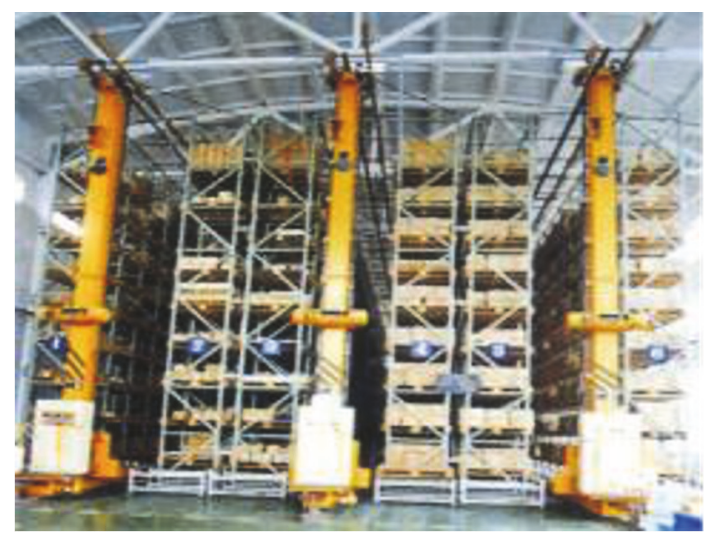

Figure 8: Stackers in State Grid Measuring Center of China.

were simulated in ASRS. In order to verify the effectiveness of the proposed model, wavelet transform and box-plot are chosen to compare with the result of PCNN-wavelet model. The results were shown as Figure 10.

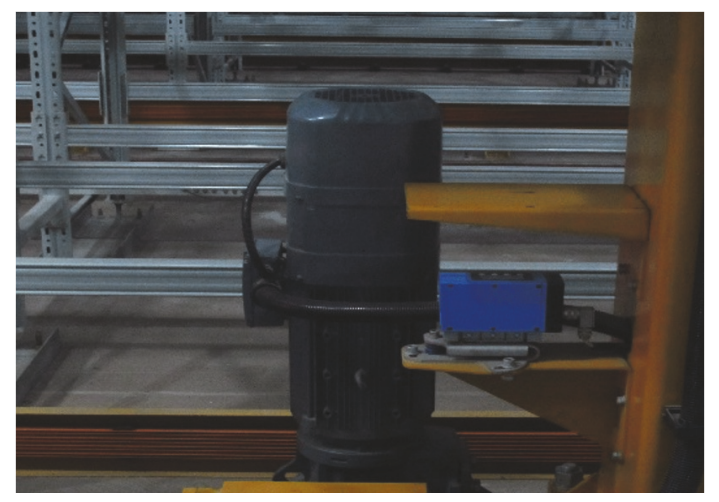

FIGURE 9: Basic structure of stacker running track.

As seen from Figure 10(a), due to the interference of noise points, it is impossible to accurately judge the abnormal points of the stacker by using only the wavelet transform. As can be seen from Figure 10(b), the abnormal 


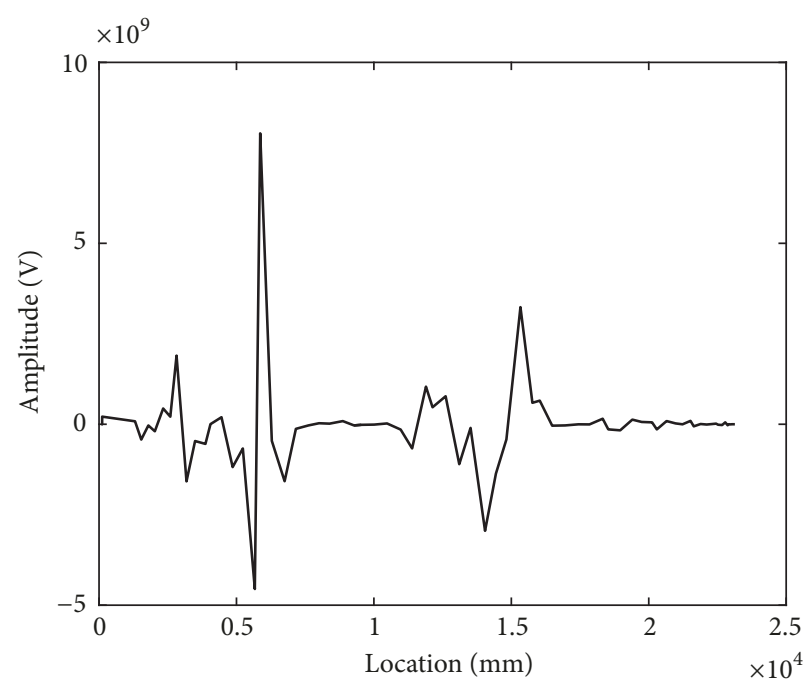

(a) Data anomaly detection results based on wavelet

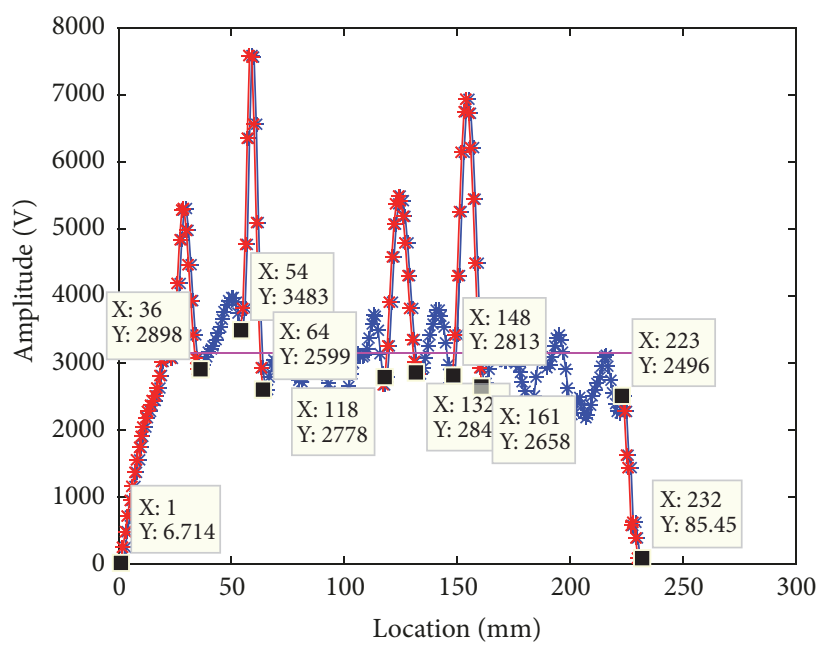

(b) Data anomaly detection results based on box-plot

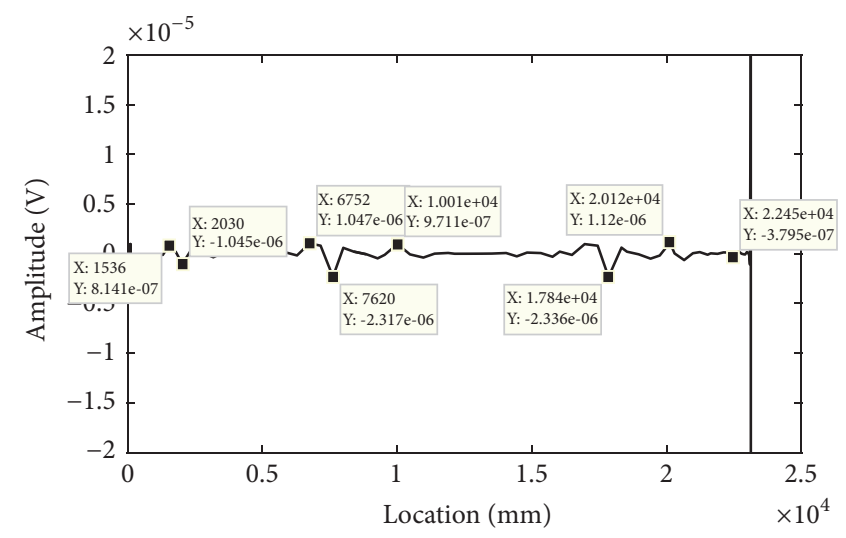

(c) Results of data anomaly detection based on PCNN-wavelet

FIGURE 10: Comparison of abnormal point detection of three methods.

point determination interval is $1 \mathrm{~m}-3.6 \mathrm{~m}, 5.4 \mathrm{~m}-6.4 \mathrm{~m}, 11.8 \mathrm{~m}$ $13.2 \mathrm{~m}, 14.8 \mathrm{~m}-16.1 \mathrm{~m}$, and $22.3 \mathrm{~m}-23.2 \mathrm{~m}$. Correspondingly, the anomaly extensions may be at $1.5 \mathrm{~m}-2.0 \mathrm{~m}, 6.7 \mathrm{~m}-7.6 \mathrm{~m}, 10 \mathrm{~m}$, $17.8 \mathrm{~m}, 20 \mathrm{~m}$, and $22.4 \mathrm{~m}$ by the judgement rules in Figure $10(\mathrm{c})$. The results can be shown as Table 2 .

Obviously, after the noise reduction of the input signal, the location of the abrupt change in the vibration signal may be depicted and described accurately by PCNN-wavelet. To ensure the operability in real engineering practice, some actual damage scenes of stacker running track were used to verify the effect of the presented algorithm. For instance, the actual testing and detecting sample photos at $1.5 \mathrm{~m}, 7.2 \mathrm{~m}$, and $18 \mathrm{~m}$ are shown in Figure 11, respectively.

In addition, there are rail welds at $10 \mathrm{~m}, 20 \mathrm{~m}$, and $22 \mathrm{~m}$, respectively.

From the experimental simulation results and the actual situation verification, although the box diagram can roughly locate the abnormal point interval, the PCNN-wavelet is more accurate and closer to the actual situation. In Figure $10(\mathrm{c})$, the simulative curve appears to be changed suddenly in these places. In other words, the cooperative
TABLE 2: Comparison of abnormal point detection results.

\begin{tabular}{lcc}
\hline Wavelet & Box-plot & PCNN-wavelet \\
\hline$\backslash$ & $1 \mathrm{~m}-3.6 \mathrm{~m}$ & $1.5 \mathrm{~m}-2.0 \mathrm{~m}$ \\
$\backslash$ & $5.4 \mathrm{~m}-6.4 \mathrm{~m}$ & $6.7 \mathrm{~m}-7.6 \mathrm{~m}$ \\
$\backslash$ & $11.8 \mathrm{~m}-13.2 \mathrm{~m}$ & $10 \mathrm{~m}$ \\
$\backslash$ & $14.8 \mathrm{~m}-16.1 \mathrm{~m}$ & $17.8 \mathrm{~m}$ \\
$\backslash$ & $\backslash$ & $20 \mathrm{~m}$ \\
$\backslash$ & $22.3 \mathrm{~m}-23.2 \mathrm{~m}$ & $22.4 \mathrm{~m}$ \\
\hline
\end{tabular}

anomaly detection method presented in this paper is effective.

In fact, the emergence of data mutation points may be caused by the depressions, cracks, and deformation at the interface in the tracks. On the other hand, the response information of the damage position may be developed to make different damage positions in different information range. And the administrators of the industrial system may locate damage positions according to numerical characteristics in the damage unit. Furtherly, the relevant departments may 


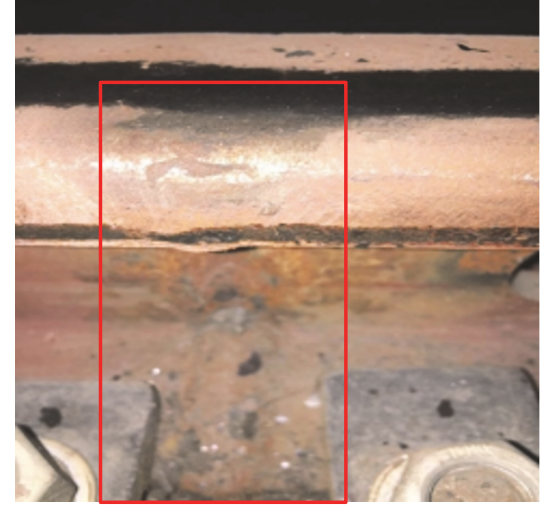

(a) Depression of lower track at $1.5 \mathrm{~m}$

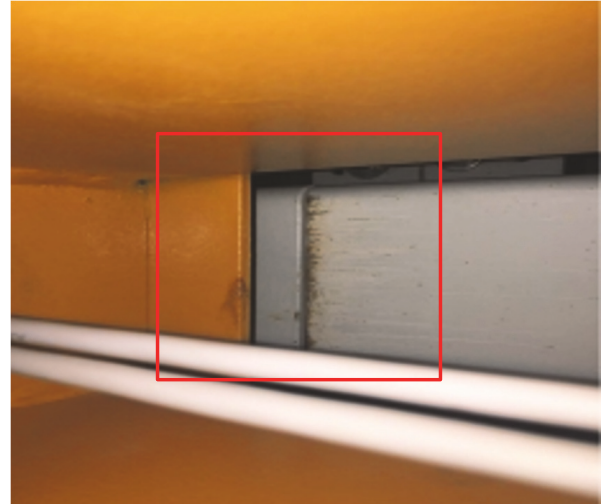

(b) Cracks appearing on upper track at $7.2 \mathrm{~m}$

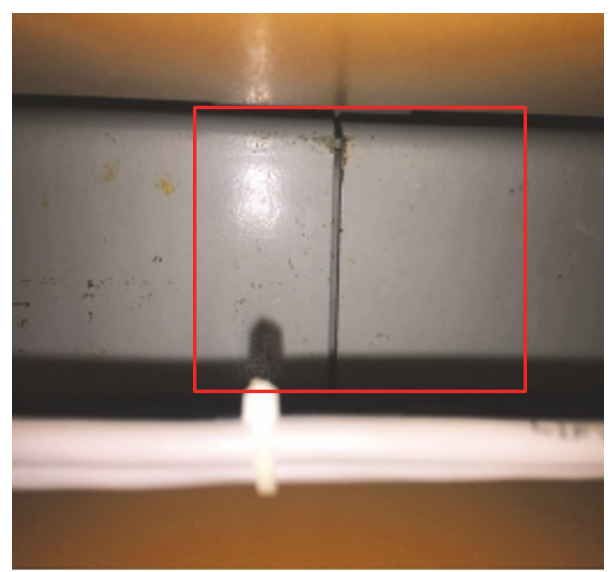

(c) Interface deformation on upper track at $18 \mathrm{~m}$

FIGURE 11: Testing and detecting sample photos from scene.

design the good maintenance strategies according to the monitoring curves. That means our algorithm may be applied to the real project.

\section{Conclusion}

In this paper, a cooperative anomaly detection method for the stacker running track in the industrial environment is presented, which is based on PCNN and wavelet transform. Firstly, the data denoising model is built based on PCNN. Then, the data is detected by wavelet transform. Finally, the rationality and validity of the proposed method are verified by example analysis and simulation. The main conclusions are as follows:

(1) In ASRS, under the influence of complex industrial environment, the data collected by the sensors will inevitably be interfered by noise, which will bring difficulties in data analysis. Simulation and real experiment examples prove that PCNN performs well in noise reduction. Moreover, it can eliminate noise interference as well as retain the original signal characteristics.

(2) After getting clean data, the wavelet transform can effectively locate the location of the abnormal point and has good applicability.
(3) After using PCNN to eliminate noise, the interference of noise-to-signal data is reduced, which is more conducive to the detection of signal data anomaly spot by wavelet transform. Through the comparison of the field and experimental data, it is shown that using PCNN and wavelet transform can realize the detection of the orbital anomaly spot of stacking machine.

\section{Data Availability}

The data used to support the findings of this study are available from the corresponding author upon request.

\section{Conflicts of Interest}

The authors declare that they have no conflicts of interest.

\section{Acknowledgments}

This work was supported by the National Natural Science Foundation of China under Grants 61703063, 61663008, 61573076, and 61004118; the Scientific Research Foundation for the Returned Overseas Chinese Scholars under Grant 2015-49; the Program for Excellent Talents of Chongqing 
Higher School of China under Grant 2014-18; the petrochemical equipment fault diagnosis Key Laboratory in Guangdong Province Foundation of China under Grant GDUPKLAB201501; the Chongqing Natural Science Foundation of China under Grant CSTC2017jcyjA1665; Science and Technology Research Project of Chongqing Municipal Education Commission of China under Grants KJ1605002, KJ1705121, KJ1705139, and KJZD-K201800701; the Program of Chongqing Innovation and Entrepreneurship for Returned Overseas Scholars of China under Grant cx2018110.

\section{References}

[1] V. Barnett and T. Lewis, Outliers in Statistical Data, John Wiley \& Sons, New York, NY, USA, 1978.

[2] M. E. Knorr and R. T. Ng, "Finding Intensional Knowledge of Distance-Based Outliers," The VLDB Journal, pp. 211-221, 1999.

[3] M. Kontaki, A. Gounaris, A. N. Papadopoulos, K. Tsichlas, and Y. Manolopoulos, "Efficient and flexible algorithms for monitoring distance-based outliers over data streams," Information Systems, vol. 55, pp. 37-53, 2016.

[4] L. Sheng, W. Zhang, and M. Gao, "Relationship between Nash equilibrium strategies and $\mathrm{H}_{2} / \mathrm{H}_{\infty}$ control of stochastic Markov jump systems with multiplicative noise," IEEE Transactions on Automatic Control, 2014.

[5] S. Ramaswamy, R. Rastogi, and K. Shim, "Efficient algorithms for mining outliers from large data sets," SIGMOD Record, vol. 29, no. 2, pp. 427-438, 2000.

[6] A. Struyf and P. J. Rousseeuw, "High-dimensional computation of the deepest location," Computational Statistics \& Data Analysis, vol. 34, no. 4, pp. 415-426, 2000.

[7] S. Zhou and W. Xu, "Deviation-based local outlier detection algorithm," Yi Qi Yi Biao Xue Bao/Chinese Journal of Scientific Instrument, vol. 35, no. 10, pp. 2293-2298, 2014.

[8] Z. Ge, Z. Song, S. X. Ding, and B. Huang, "Data Mining and Analytics in the Process Industry: The Role of Machine Learning," IEEE Access, vol. 5, pp. 20590-20616, 2017.

[9] H. Liu, X. Li, and J. Li, "Efficient Outlier Detection for HighDimensional Data," IEEE Transactions on Systems Man \& Cybernetics Systems, no. 99, pp. 1-11, 2017.

[10] M. Markou and S. Singh, "Novelty detection: a review-part 2: neural network based approaches," Signal Processing, vol. 83, no. 12, pp. 2499-2521, 2003.

[11] J.-S. Wang and J.-C. Chiang, "A cluster validity measure with outlier detection for support vector clustering," IEEE Transactions on Systems, Man, and Cybernetics, Part B: Cybernetics, vol. 38 , no. 1, pp. 78-89, 2008.

[12] S. Mallat and W. L. Hwang, "Singularity detection and processing with wavelets," IEEE Transactions on Information Theory, vol. 38, no. 2, pp. 617-643, 1992.

[13] W. P. Stuart-Bruges and V. Srinivasan, "Self-diagnostic control of a stacker crane," Electronics and Power, vol. 21, no. 2, pp. 108111, 1975.

[14] Z. Ge, "Review on data-driven modeling and monitoring for plant-wide industrial processes," Chemometrics and Intelligent Laboratory Systems, vol. 171, pp. 16-25, 2017.

[15] LI. Xiaoping and YU. KangKang, "Research on Key Technology in Remote Fault Diagnosis of the Stacker," Journal of Lanzhou Jiaotong University, vol. 30, no. 4, pp. 15-20, 2011.
[16] H. Darong, T. Jianping, and Z. Ling, "A fault diagnosis method of power systems based on gray system theory," Mathematical Problems in Engineering, vol. 2015, Article ID 971257, 2015.

[17] K. Zhang, K. Peng, and J. Dong, "A Common and Individual Feature Extraction-Based Multimode Process Monitoring Method With Application to the Finishing Mill Process," IEEE Transactions on Industrial Informatics, vol. 14, no. 11, pp. 48414850, 2018.

[18] K. Zhang, H. Hao, Z. Chen, S. X. Ding, and K. X. Peng, "A comparison and evaluation of key performance indicator-based multivariate statistics process monitoring approaches," Journal of Process Control, vol. 33, pp. 112-126, 2015.

[19] H. Darong, K. Lanyan, M. Bo, Z. Ling, and S. Guoxi, "A New Incipient Fault Diagnosis Method Combining Improved RLS and LMD Algorithm for Rolling Bearings With Strong Background Noise," IEEE Access, vol. 6, pp. 26001-26010, 2018.

[20] W. Jianguo, Y. Haipeng, and Z. Wenxing, "Data noise reduction method based on modified PCNN," in China Measurement Test, vol. 42, pp. 92-95, 2016.

[21] Z. Wenxing, Y. Haipeng, and W. Jianguo, "Research on Data Noise Reduction Method Based on Modified PCNN," Machinery Design Manufacture, vol. 2, pp. 25-28, 2015.

[22] R. Eckhorn, H. J. Reitboeck, M. Arndt, and P. Dicke, "Feature Linking via Synchronization among Distributed Assemblies: Simulations of Results from Cat Visual Cortex," Neural Computation, vol. 2, no. 3, pp. 293-307, 1990. 


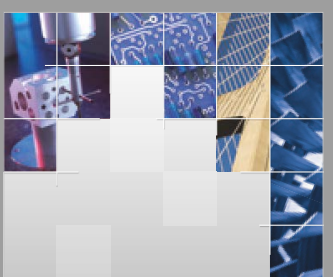

\section{Enfincering}
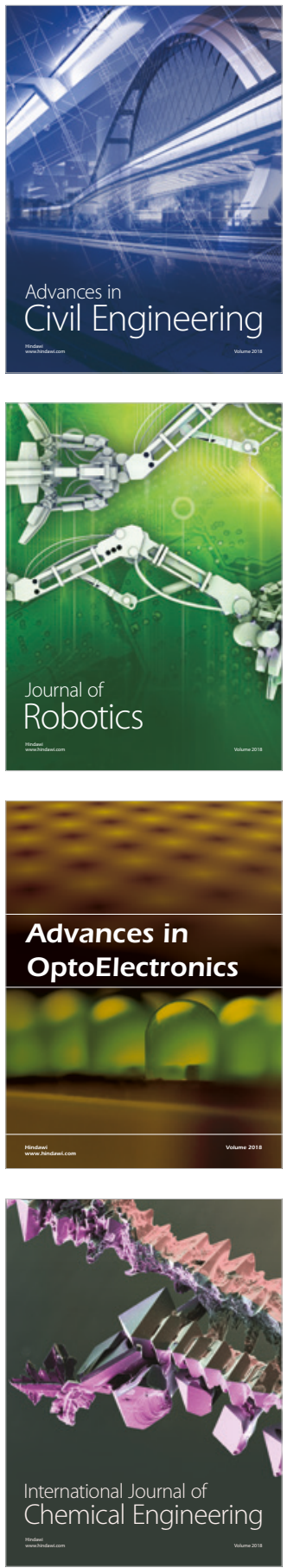

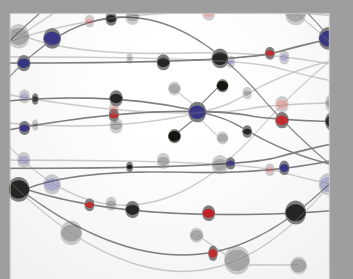

\section{Rotating \\ Machinery}

The Scientific World Journal

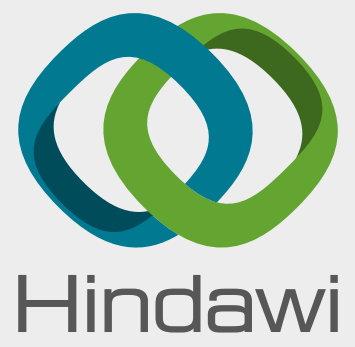

Submit your manuscripts at

www.hindawi.com
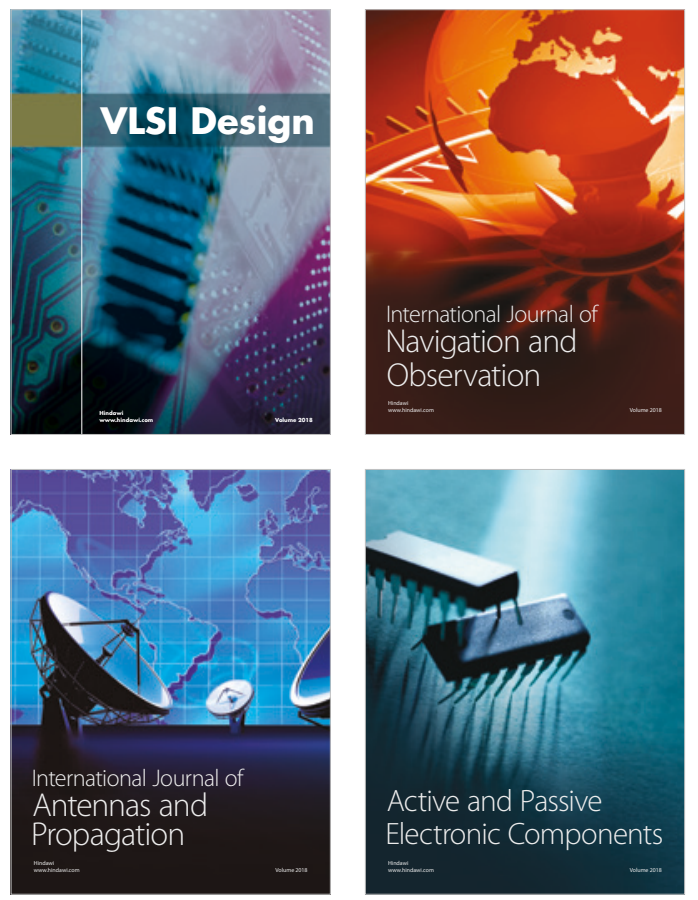
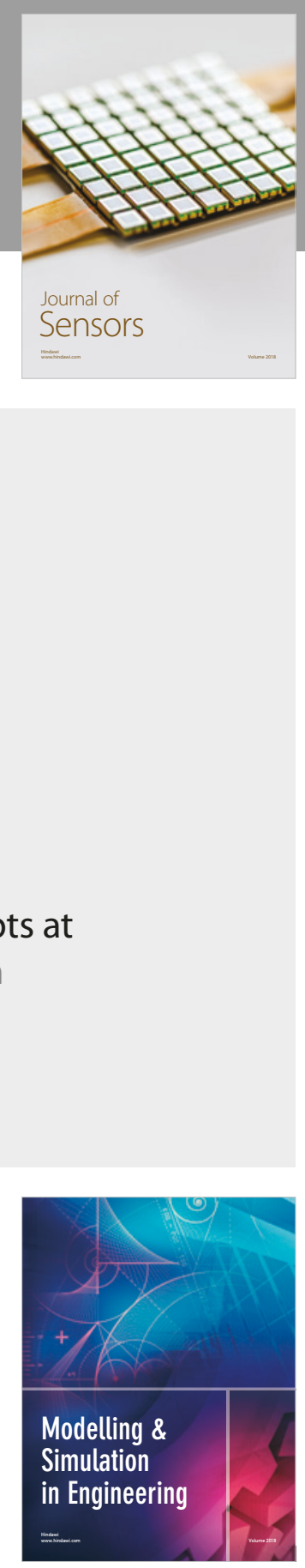

\section{Advances \\ Multimedia}
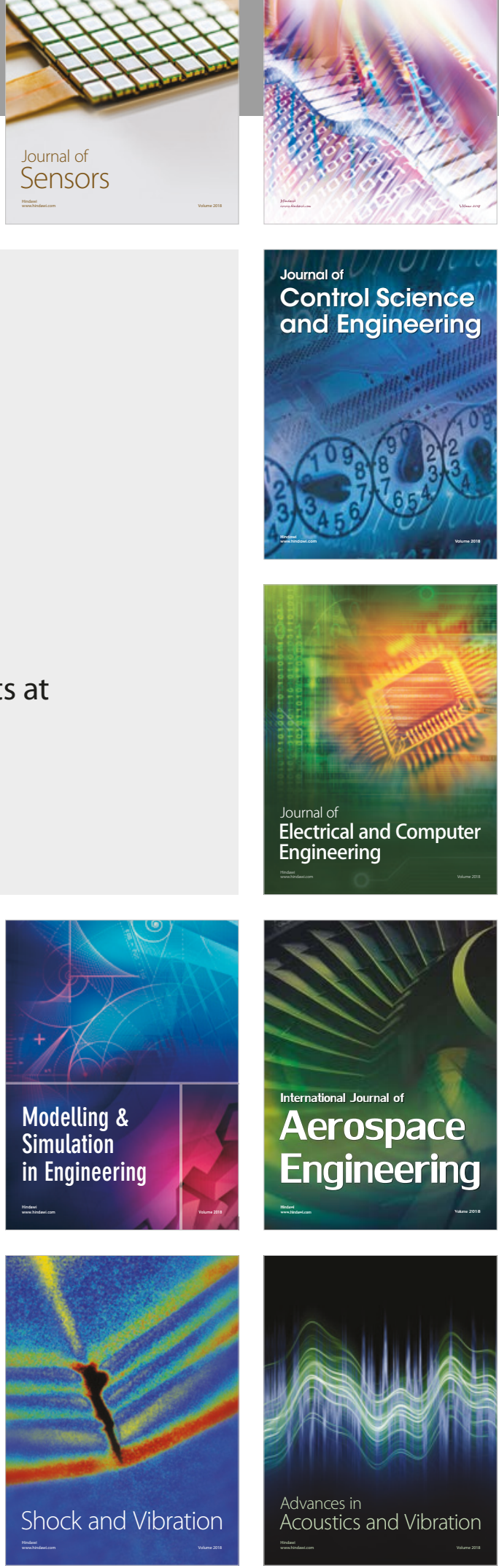